\title{
A Novel Vertical Handoff Algorithm Based on Differential Pre- Decision and Improved Utility-Function Method
}

\author{
Chenghai ${ }^{1}{ }^{l}$, Dawei Ma ${ }^{1 *}$, Feng Wang ${ }^{l}$ and Xiaoan Bao ${ }^{l}$ \\ Zhejiang Sci-Tech University \\ ych@zstu.edu.cn
}

\begin{abstract}
The paper proposes a vertical handoff algorithm based on differential pre-decision and improved utility-function method. This algorithm is relied on utility function to improve its two parameters, which are received signal strength and bandwidth, by using their normalized values to determine the target network. Then, it enters judgment stage of vertical handoff, where introduce differential prediction algorithm that has good accuracy to further constraint the switching decision through taking a prediction of the RSS into account. The purpose is to reduce the impact of ping-pong effect and to obtain accurate handoff. The paper applies MATLAB software to gain the simulated analysis based on this algorithm, And compares the performance of this switching algorithm with utility function algorithm to demonstrate its priority. Simulation results shows that the proposed algorithm can significantly reduce the impact of the ping-pong effect and improve the switching efficiency and the system performance as well in heterogeneous network environments.
\end{abstract}

Keywords: Heterogeneous network environments, Vertical handoff, Pre-decision algorithm, Utility-function

\section{Introduction}

The features of the next-generation mobile communication systems are that multiple wireless access technologies coexist, complement with each other and integrate seamlessly. Different access technologies differ in bandwidth, transmission delay, the mobility of coverage, etc. It is evident that any single existing wireless and mobile network fails to get the requirements of the wide coverage, low latency, high bandwidth, low cost [1-3]. UMTS system provides wide-area wireless coverage and supports the global roam for mobile communications, having voice services and the low-speed data services. WLAN provides hotspot coverage, supports nomadic mobility, and has high data transferring rate. However, due to the limited scope of WLAN coverage and the lack of roaming and mobility support, it is desirable to access a wide range of cellular network coverage, such as Universal Mobile Telecommunications System (UMTS). They could complement highly with each other and make up for their own shortcomings. [4] When the multi-mode terminal equipped with heterogeneous network interfaces move in overlapping coverage areas of the two networks, it could choose the appropriate access network based on business and load conditions. It requires seamless handoff between heterogeneous networks to ensure continuous service delivery, and finally provide users with a better service experience.

Aiming to make the mobile terminal move from one network access point to another with continuous connection, seamless roam in heterogeneous networks environments, have proposed vertical handoff technique. Many documents have proposed various vertical handoff decision algorithms. In Ref [3], by using the fuzzy multi-properties decision-making problem judgment to describe the vertical handoff decision, two methods: SAW (Simple weighting method), and TOPSIS (close to the ideal solution for 
ordinal preference methods) are successfully proved and proposed. In Ref [4], the cost function introduced AHP algorithm uses the analytic hierarchy process to determine the relationship of weight between variety of properties, construct comparative judgment matrix and testing its consistency, and then use the cost function to judge the switching of vertical handoff. In Ref [11], the normalized bandwidth and signal strength of a simple linear weighted is defined as the cost function to improve the performance of vertical handoff. Ref [12] presents a new architecture for network convergence, which allows the mobile station perform in parallel data transmission on the WLAN and voice transmission on UMTS to ensure the high transfer rate of data services and the continuity of voice services. But only the received signal strength (RSS) as an indicator of vertical handoff algorithm cannot meet the system requirements.

This paper presents a novel utility function with two normalized parameters and proposes differential pre-decision vertical handoff algorithm. The algorithm is based on utility function, making some improvements on the bandwidth and the signal strength of these two parameters, using their normalized values to determine the target network. What' s more, it enters the decision stage of vertical handoff. In this stage, it introduces differential predictive algorithm before actual handoff decision to filter out users with high mobility or low RSS from using the WLAN. The purpose is to reduce the impact of the ping-pong effect as well as improve the performance of handoff.

\section{Utility Function}

In heterogeneous network environment, the vertical handoff decision problem between different network is multi-Properties decision-making problem. Thus, for the improved algorithm, network performance is still based on the value of the utility function to determine.

\subsection{General Utility Function}

In selecting the parameters of the utility function, some researchers select signal strength and bandwidth, which may be expressed by Equation (1.1)

$$
y_{n}=\omega_{B} \times \ln \left(B_{n}\right)+\omega_{R S S} \times \ln \left(R S S_{n}\right)
$$

Where $\omega_{B}$ is the weight of network bandwidth and $\omega_{R S S}$ is the weight of network signal strength, The constraint between $\omega_{B}$ and $\omega_{R S S}$ is given by:

$$
\omega_{B}+\omega_{R S S}=1
$$

And the available bandwidth ${ }^{B_{a}}$ of the network satisfies the formula above:

$$
B_{a}=B_{t}-B_{o}
$$

Where ${ }^{B_{t}}$ is the total network bandwidth, and ${ }^{B}$ is network bandwidth of users. Thus, the utility function expression as (1.4):

$$
y_{n}=\omega_{B} \times \ln (B)+\omega_{R S S} \times \ln (R S S)
$$

Where $R S S, \omega_{B}, \omega_{R S S}$ are the received signal strength of network, the weight of network bandwidth and the weight of network signal strength ,respectively. 


\subsection{Improved Utility Function}

General utility function is different with improved utility function in the parameters. Improved utility function uses improved bandwidth and RSS. The reason to do that is determined by the nature of network, and the purpose is to trade off the performance of each network. Improved utility function select the normalized signal strength and bandwidth utilization.

(1) Normalized Bandwidth

Bandwidth utilization is the bandwidth normalized, i.e. the ratio of available bandwidth to the total bandwidth of the current network. Clearly, the greater its value is, the higher data transfer rate can be obtained, and the smaller the transmission delay is, finally the more difficult the network congestion will be. The reason why in this paper the bandwidth and the signal strength is normalized is that the range of the maximum bandwidth and effective available bandwidth provided by WLAN and UMTS networks are different. This may cause a lower switching efficiency. Therefore, in the vertical handoff decision process, in order to ensure fairness and effectiveness after switching, it select bandwidth utilization as a parameter of target network switching judgment. The utilization is defined as following:

$$
B_{i}=\frac{B_{a}}{B_{t}}
$$

where $B_{a}$ is the available bandwidth, and $B_{t}$ is the total bandwidth. As a result, larger $B_{i}$ implies lower traffic load.

(2) Normalized Rss

In general, the greater the received signal strength is, the better quality of the network is, therefore the received signal strength of network is more acceptable for the mobile terminal. However, in a heterogeneous network, it is difficult to compare the received signal strength. For different wireless networks have different maximum transmission power and receiver threshold. Thus the relative received signal strength can be used to compare different types of wireless and mobile networks in heterogeneous networks. Furthermore, the RSS normalized. We generally use the receiver threshold and the

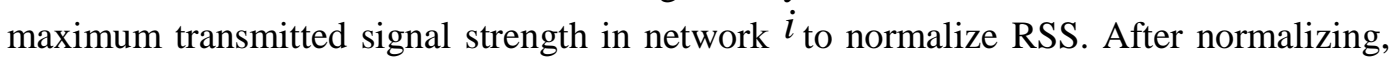
RSS is defined in $i$ network as following:

$$
S_{i}=\frac{P_{i}^{c}-P_{i}^{t h}}{P_{i}^{\max }-P_{i}^{t h}}
$$

where $P_{i}^{c}$ is the current received signal strength from network $i, P_{i}^{\text {th }}$ is the receiver threshold in network $i$, and $P_{i}^{\max }$ is the maximum transmitted signal strength in network $i$. $S_{i}$ is normalized values for RSS. In order to assess the performance of handoff algorithm, we should take the channel model and mobile model into account. In analysis architecture, It uses typical Log-linear path loss model, and considers the effect that shadow fading influence on the received signal strength. RSS is represented as a function whose Parameters have the distance (d) between the mobile station and the access point

$$
P_{i}^{c}=P_{i}^{\max }-L-10 n \log (d)-X_{\sigma}
$$

Where $P_{i}^{\max }$ is the maximum transmitted signal strength in network; $L$ is constant signal power loss; ${ }^{n}$ is the path loss exponent, and its value is typically between 2 to 4 ; 
$X_{\sigma}$ is shaded attenuation, satisfying Gaussian random distribution with mean 0 and standard deviation $\sigma$. Above, the utility function is set as:

$$
y=\omega_{B} \times \ln \left(B_{i}\right)+\omega_{R S S} \times \ln \left(S_{i}\right) \quad 1.8
$$

Where ${ }^{B_{i}}$ represents the network bandwidth availability, $S_{i}$ express RSS normalized value, $\omega_{B}$ is the weight of network bandwidth and $\omega_{R S S}$ is the weight of the received signal strength, and they two satisfy the equation (1.2) .

\section{The Introduction of Differential Pre-Decision to Vertical Handoff Algorithm}

Before performing a vertical handoff, it introduces a differential prediction algorithm, adding a constraint, and this condition can be described as a decision that based on the prediction of the received signal strength. When the condition is satisfied, then it would switch. Through this way, the number of switches will be significantly improved, and the ping-pong effect can be improved. Here we will discuss about the differential prediction algorithm and model of pre-decision.

\subsection{Differential Prediction Algorithm}

In the stage of discovering target network, it uses the value of the utility function to determine the target network, then, it enters the decision stage. Considering the prediction of signal strength as the decision parameters can improve the speed and accuracy of switching. But it is difficult to achieve complex prediction of signal strength for the mobile terminal. Thus in this paper, we use a simple forward differential prediction method to carry out the decision by predicting the next time received signal strength of the network. In this paper, the signal predictor formula is above:

$$
\hat{r}(k+1)=2 r(k)-r(k-1)
$$

Where $k$ is the current time; $r(k)$ is the input signal strength value at $k$ time, $r(k-1)$ is the input signal strength value at $k-1$ time. It can be seen from Figure 1 , and this simple prediction algorithm can get better forecasting results, which is a practical and effective method. 


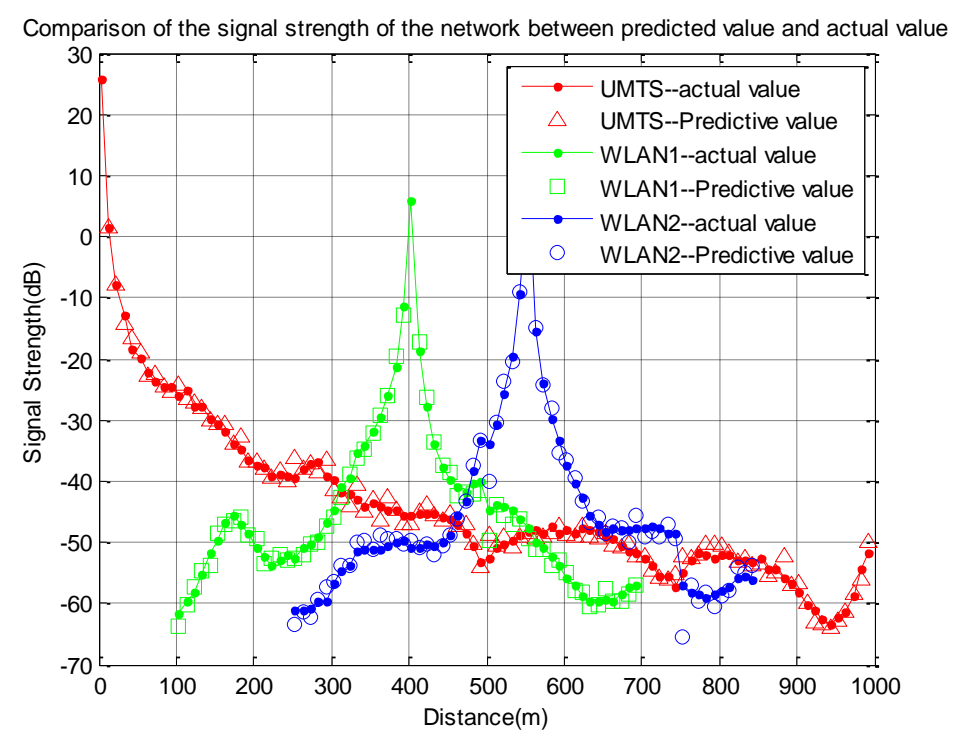

Figure 1. Prediction Signal (PR) And Actual Signal (CR)Curve

\subsection{Pre-Decision Model}

Adding the pre-decision before actual handoff decision is to reduce those unnecessary handoff and the ping-pong effect, improve performance and effectiveness of vertical handoffs. This stage can be summarized as a constraint that if the predicted signal strength of current network and target network were within the pre-decision threshold, then switching occurs. Conversely, no switching occurs. In WLAN network, $P_{r W}$ is predecision threshold of RSS; $P_{r U}$ is pre-decision threshold of RSS in UMTS network. Since the switching from WLAN to UMTS and from UMTS to WLAN are symmetrical process, so only pre-handoff decision process from the UMTS to the WLAN is given. Shown in Figure 2:

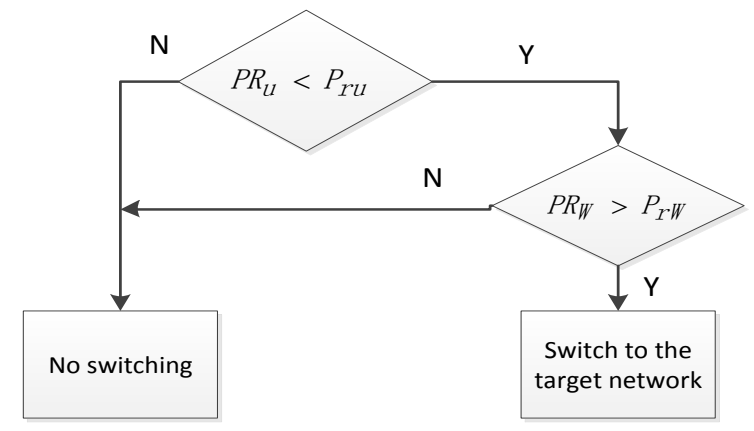

Figure 2. Pre-Decision Process

\section{Simulation}

Author names and affiliations are to be centered beneath the title and printed in Times New Roman 12-point, non-boldface type. Multiple authors may be shown in a two or three-column format, with their affiliations below their respective names. Affiliations are centered below each author name, italicized, not bold. Include e-mail addresses if possible. Follow the author information by two blank lines before main text. 


\subsection{Heterogeneous Network Environment Settings}

Environmental settings is shown as Figure 3. The mobile terminal starts to move from the designated position in UMTS and WLAN coverage area, and across heterogeneous network cell. Thus, In order to meet their own needs for mobile users, it will happen network switching in this area.

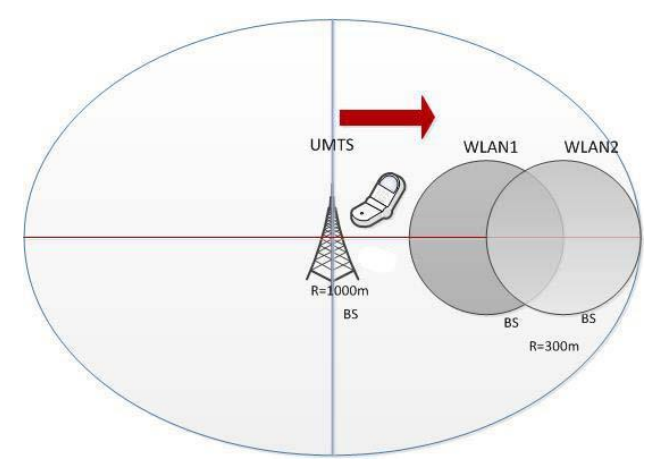

Figure 3. Heterogeneous Network Convergence Model

(1) Business Type Parameter

It is set that the starting place of mobile terminal randomly generate in this overlap area, and generally the speed of mobile terminal is between $1 \mathrm{~m} / \mathrm{s}$ and $50 \mathrm{~m} / \mathrm{s}$. It is linear motion. Typically, the service type is set in the following table 1 :

Table 1. Appsoccupied Bandwidth and Real-Time Requirements

\begin{tabular}{lll}
\hline Type of Service & Bandwidth & Real-time \\
\hline Voice Session & $0.38 \mathrm{M}$ & Real time \\
Data Download & $2 \mathrm{M}$ & Non-real time \\
\hline
\end{tabular}

(2) Network Performance Parameters

Network performance parameters is shown as Table 2:

Table 2. Network-Related Parameters

\begin{tabular}{|c|c|c|c|}
\hline Environment Settings & UMTS & WLAN1 & WLAN2 \\
\hline total network bandwidth & 5 & 16 & 16 \\
\hline $\begin{array}{l}\text { The maximum } \\
\text { transmitted } \\
\text { station }(\mathrm{dBm})\end{array}$ & 45 & 20 & 20 \\
\hline
\end{tabular}

\begin{tabular}{llll}
$\begin{array}{c}\text { Transmitting } \\
\text { gain }\end{array}$ & 1 & 1 & 1 \\
Receive antenna gain & 1 & 1 & 1 \\
Receiver threshold & -80 & -80 & -80 \\
Radius(m) & 1000 & 300 & 300 \\
Carrier frequency & 2300 & 2500 & 2500 \\
\hline
\end{tabular}

(3) Other Relevant Parameters Setting 
For other parameters setting, This article will set coordinate about UMTS and WLAN base stations to $(0,0),(400,0)(555,0)$. Initially, the speed of mobile terminal is set to $4.5 \mathrm{~m} / \mathrm{s}$, and its move is a uniform linear motion. The mobile terminal scan the network at interval $0.2 \mathrm{~s}$. The weight of $\omega_{B}$ and $\omega_{R S S}$ in the utility function are set to equal value with 0.5 .

\subsection{Simulation Results and Performance Analysis of Algorithm}

\subsubsection{Improved Vertical Handoff Algorithm Simulation Results Based on the Utility Function}

Assuming that the speed of the mobile terminal is $4.5 \mathrm{~m} / \mathrm{s}$; WLAN cell range is $300 \mathrm{~m}$; UMTS cell range is $1000 \mathrm{~m}$; the distance between WLAN1 access point and UMTS base stations is $400 \mathrm{~m}$, and the distance between of WLAN2 UMTS base stations and access points is $555 \mathrm{~m}$. With the movement of mobile terminal in the overlay network, the changing curves of current RSS are shown in Figure4 and Figure5. Figure4 and Figure5 is obtained respectively by the utility function simulation and the improved utility function simulation.
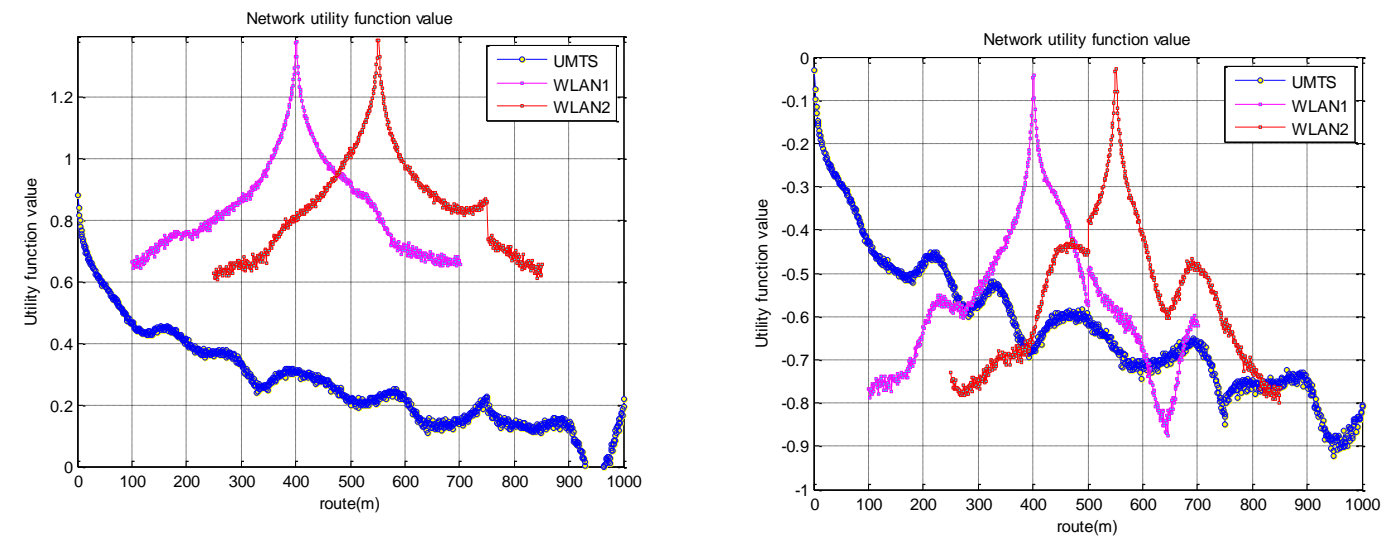

Figure 4. Utility Function Value

Figure 5. Improved Utility Function Value

The simulation results can be seen from Figure 5 that the utility function value has the maximum, so the network performance is optimal in the vicinity of the respective base stations. Since this figure uses the improved utility function, simulation results that are get by comparing with the results original utility function shows that the utility function value is not always largest after entering the scope of network. It changes alternately, which is in line with the actual situation. 


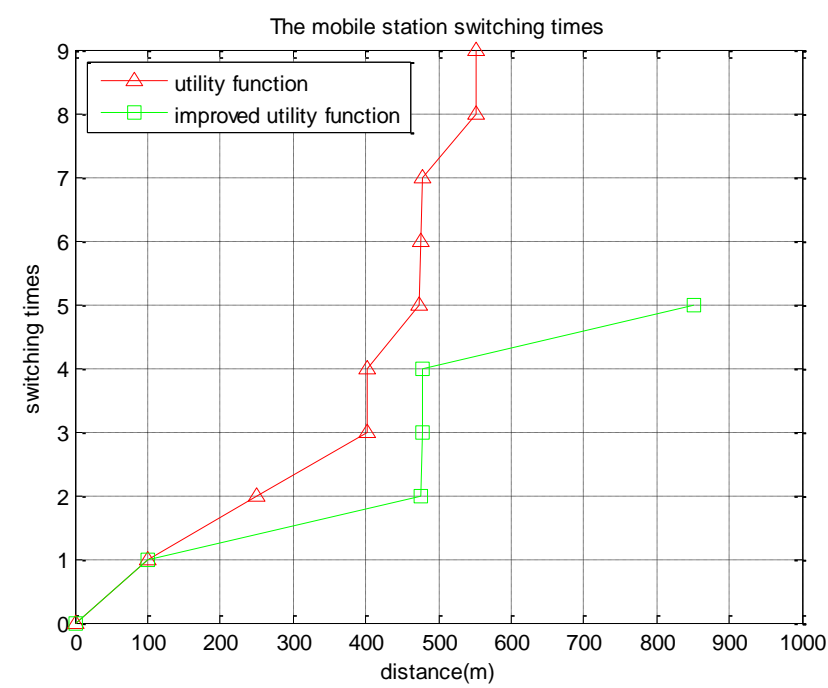

Figure 6. Two Kinds of Handoff Decision Algorithms Compare Switching Times

Figure 6 is the times of the vertical handoff, which is gotten by the use of utility function and improved utility functions. It can clearly be seen from Figure 6 that the improved utility function significantly reduce the number of vertical switching, and also improve switching over the transition zone.

\subsubsection{The Simulation Results of the Introduction of Pre-Decision Vertical Cut Algorithm Based on the Utility Function}

In simulation environment, we set the parameters as $P_{r w}=-40 \mathrm{~dB}, P_{r u}=-42 \mathrm{~dB}$, the comparison of the times of switching is shown in Figure 7. While Figure 7 shows that the pre-decision vertical handoff algorithm based utility function significantly reduce switching frequency, which is not possible with several other algorithms. Pre-decision algorithm has a pre-decision process, if the predicted signal strength values of current network and target network are within the pre-decision threshold, then switching occurs. Conversely, no switching occurs. Thus, the number of switches are significantly reduced, what is more, the switching efficiency and the ping-pong effect are largely improved.

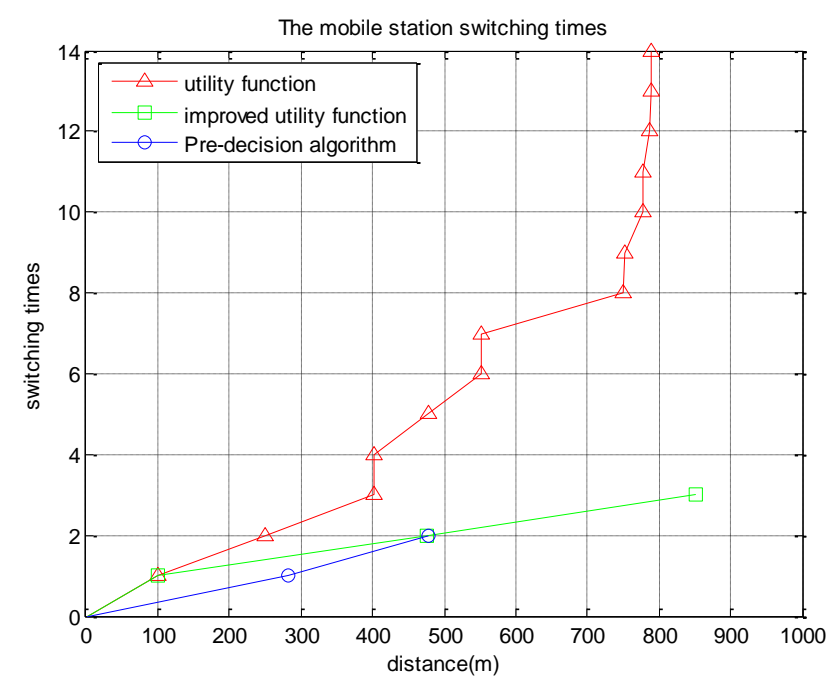

Figure 7. Three Kinds of Handoff Decision Algorithms Compare Switching Times 


\section{Conclusion}

Firstly, the paper starts from the utility function, its parameters are normalized improvements, and the simulation results were compared with the traditional utility function algorithm, getting the advantages of the former algorithm. Secondly, it is based on the improved utility function, and added the pre-decision of this process, that is the use of a predictive value of the signal strength of constraints, and compare this algorithm with the first two to get the outcome; By contrast with the previously mentioned several common handoff decision algorithm analysis, the simulated results of its performance shows great advantages, furthermore, it proves that the ping-pong effect of the algorithm as well as the performance of the system have been greatly improved.

\section{Acknowledgements}

This work is supported by the Natural Science Foundation of Zhejiang Province, China (Grant No. LY12F02041), the National Natural Science Foundation of China (Grant No. 61374020 and 61379036), Funded by Zhejiang Top Priority Discipline of Textile Science and Engineering \& Engineering Research Center of Clothing Technology of Zhejiang Province.

\section{References}

11] R.H. Katz and E.A. Brewer, "The case for wireless overlay networks", Proceedings of SPIE Multimedia and Networking Conference, (1996), pp.77-88, San Jose, CA.

[2] 李军. 异构无线网络融合理理论与实现. 电子工业出版社, (2009).

[3] W. Zhang, "handoff decision using fuzzy MADM in heterogeneous networks. IEEE Wireless Communications and Networking Conference”, WCNC'04, vol. 2, (2004), pp. 653-658, Atlanta, GA.

[4] 㚞辰曦,俞成海,何永伟. 一种改进的的基于代价函数的垂直切换算法.浙江理工大学学报, (2014) 年 09 期。

[5] E. Stevens-Navarro and V.W.S. Wong, "Comparison between vertical handoff decision algorithms for heterogeneous wireless networks [C]", Vehicular technology conference, 2006. VTC 2006-Spring. IEEE 63rd. IEEE, vol. 2, (2006), pp. 947-951.

[6] S. Milena and M. Petri, "Algorithmic approaches for vertical handoff in heterogeneous wireless environment", Proceeding of IEEE WCNC2007, (2007), pp. 3783-3788, Kowloon China.

[7] Q.-Y. Song and A. Jamalipour, "Network selection in an integrated wireless LAN and UMTS environment using mathematical modeling and computing techniques", IEEE Wireless Communication, vol. 12, no. 3, Jun. (2005), pp. 42-48.

[8] X. Liu, L.G. Jiang and C. He, "A novel fuzzy logic vertical handoff algorithm with aid of differential prediction and pre-decision method", Procedeing of IEEE ICC'06, (2006), pp. 5665-5670, Scotland.

[9] M. Stemm and R.H. Katz, "Vertical handoffs in wireless overlay networks", ACM Mobile Networking and Applications (MONET), vol. 3, no. 4, (1998), pp.335-350.

[10] T. Fatma and S. Muhammed, "On the vertical handoff decision for wireless overlay networks", Proceeding of the seventh IEEE International Symposium on Computer Networks (ISCN'06, (2006), pp. 111-115, Turkey.

[11] W. Shen and Q.A. Zeng, "Cost-function-based network selection strategy in integrated wireless and mobile networks [J]", Vehicular Technology, IEEE Transactions on, vol. 57, no. 6, (2008), pp. 37783788.

[12] M. Jaseemuddin, "Architecture for integrating UMTS and 802. 11 WLAN networks [c]”, Proceeding of the 8th ISCC, (2003), pp. 716-723, Turkey: IEEE ISCC. 


\section{Author}

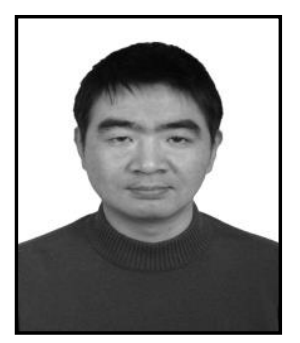

Chenghai Yu, Male (1975-), Zhejiang Sci-Tech University, associate Professor, Graduated from Southwest Jiaotong University, Main research: Network Communications, Mobile Application Development.

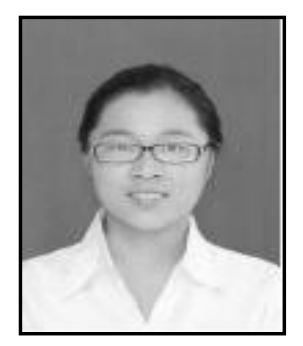

Dawei Ma, Female (1990-), Zhejiang Sci-Tech University, master, Main research: Computer Network and Application Development.

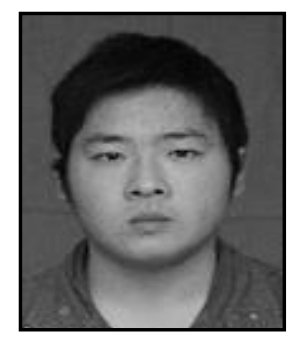

Feng Wang, Male, native place:jiangsu. The graduate student of Zhejiang Sci-Tech University. Main research: the Computer Science and Technology

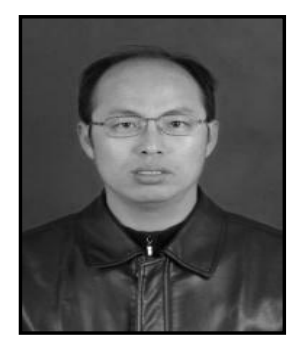

Bao Xiao'an (1973), Zhejiang Sci-Tech University, Professor, main research: adaptive software, software testing and intelligent information processing. 